\title{
Editorial: New Editorial Board Members
}

THOMAS G. DIETTERICH

tgd@cs.orst.edu

Executive Editor

As the field of machine learning has grown and matured, the number of active, senior researchers in the field has grown as well. Our journal faced a problem: If we added all of these excellent scientists to the editorial board, it would soon become large and unwieldy. Hence, at the 1993 meeting of the editorial board, it was decided to develop a system of rotating 3-year terms, so that over time a wide range of researchers in machine learning would have the opportunity to serve on the editorial board and contribute to the quality and success of the journal.

To implement this rotating-term system, currently-serving editorial board members were randomly assigned to one of three groups with terms ending December 31, 1993, December 31, 1994, and December 31, 1995. I want to take this opportunity to recognize and thank those board members whose terms expired December 31, 1993:

Saul Amarel, John Anderson, Geoff Hinton, Pat Langley, Doug Lenat, Tom Mitchell, Jack Mostow, Roger Schank, and Patrick Winston.

Each of these scientists was a founding member of the editorial board for Machine Learning, and the journal has benefitted greatly from their participation and support. I particularly want to cite Pat Langley, Tom Mitchell, and Jack Mostow for their contributions as editors.

To fill these vacancies, the editorial board elected the following new editorial board members, who will serve until December 31, 1996:

Ivan Bratko, Wray Buntine, William Cohen, Michael Jordan, Leslie Kaelbling,

Michael Kearns, Pat Langley, Tom Mitchell, Steve Muggleton, Michael Pazzani,

Gerry Tesauro, Rob Schapire, Kurt VanLehn

On behalf of the readership of Machine Learning, I want to warmly welcome these new editorial board members and thank them for their willingness to serve.

The observant reader will also note that the list of action editors for the journal has changed. In the past year, four action editors have stepped down: Dennis Kibler, Bruce Porter, and Rich Sutton all completed three-year terms; Steve Minton resigned to take the position of Executive Editor of the Journal for Artificial Intelligence Research. Each of these action editors has put in hundreds of hours of volunteer time to help make this journal a success, and I thank each of them for their dedication.

To fill these vacancies, the editorial board elected Andrew Barto, Jude Shavlik, and Douglas Fisher to new three-year terms as action editors. They join two other new action editors who were elected in 1993: Raymond Mooney and Lorenza Saitta. Each of these new action editors has already provided great assistance to the journal through their 
prompt and insightful reviews of submitted papers. I am extremely pleased that they have taken on the added responsibility of coordinating the review process for Machine Learning manuscripts. 